\title{
Special functions, transcendentals and their numerics *
}

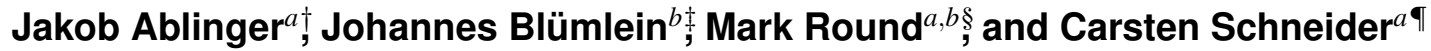 \\ a Research Institute for Symbolic Computation (RISC), Johannes Kepler University, \\ Altenberger Straße 69, 4040, Linz, Austria \\ ${ }^{b}$ Deutsches Elektronen-Synchroton (DESY), Platanenalle 6, D-15738, Zeuthen, Germany
}

Cyclotomic polylogarithms are reviewed and new results concerning the special constants that occur are presented. This also allows some comments on previous literature results using PSLQ.

13th International Symposium on Radiative Corrections (Applications of Quantum Field Theory to Phenomenology)

25-29 September, 2017

St. Gilgen, Austria

${ }^{*}$ This work was supported in part by the Austrian Science Fund (FWF) grant SFB F50 (F5006-N15, F5009-N15) and the European Commission through contract PITN-GA-2012-316704 (HIGGSTOOLS).

$\dagger$ jablinge@ risc.uni-linz.ac.at

$\ddagger$ Johannes.Bluemlein@desy.de

$\S_{\text {speaker, mark.round@desy.de }}$

『cschneid@ risc.uni-linz.ac.at 


\section{Outline}

Through the usual procedure of introducing Feynman (or Schwinger) parameters one can perform the momentum integrals of Feynman diagrams. This leads to multiply nested integrals over $(0,1)$. In principal one should not expect such integrals to always be expressible in terms of elementary and known special functions. Immediately then one must ask what it means to 'solve' a Feynman diagram. To compare theoretical results to experimental data a solution could be interpreted as a form which can be evaluated quickly. On mathematical grounds, a solution could be interpreted as expressing integrals in terms of a set of basis objects which admit no further relations amongst them. Naturally one would like to achieve both goals simultaneously. Perhaps the most basic set of objects, beyond elementary and known special functions, found in particle physics are the harmonic polylogarithms [1] or, in Mellin space, the harmonic sums [2]. The harmonic polylogarithms are a generalisation of the classical polylogarithm, see e.g. [3] and the Nielsen integrals [4]. These harmonic polylogarithms generalise to the cyclotomic polylogarithms [5] and then to the generalised (or Goncharov) polylogarithms and beyond, see for example [5-8] for a literature. ${ }^{1}$ The questions are what relations exist between these polylogarithms and how can they be efficiently evaluated?

All of these types of polylogarithms are defined through iterated integrals ${ }^{2}$

$$
H(\vec{a} ; x):=\int_{0}^{x} d t f_{a_{1}}(t) H\left(\vec{a}_{i>1} ; t\right), \quad H(\emptyset ; x):=1 .
$$

Here the vector notation $\vec{a}$ is something of an abuse of notation as $\vec{a}$ will be frequently treated as an ordered list. Meanwhile $\vec{a}_{i>1}$ refers to $\vec{a}$ with the first element removed. The components of $\vec{a}$ are known as indices and the length of $\vec{a}$ is known as the weight of the polylogarithm. Making a choice for the functions $f_{i}$ one falls into the various classes of polylogarithms. All of the classes used in particle physics include $f_{i}(t)=0$ which necessitates an additional definition for the weight $n$ polylogarithm whose indices are all zero, $H(\overrightarrow{0} ; x):=\frac{1}{n !} \log ^{n} x$. The treatment here will be largely formal and little further attention will be given to questions of existence.

Iterated integrals have several properties on general grounds [14-16]. There are identities from using integration by parts and from the shuffle product. The shuffle of two lists $a$ and $b$ can be defined recursively as follows,

$$
a \amalg \emptyset:=\{a\}, \quad \emptyset \amalg b:=\{b\}, \quad a \amalg b:=\widehat{a_{1}}\left(a_{i>1} \amalg b\right) \cup b_{1}^{\curlyvee}\left(a \amalg b_{i>1}\right) .
$$

Here $a_{1}^{-} X$ is the list of lists $X$ with $a_{1}$ pre-pended to each element of $X$. As defined here the result of $a \amalg b$ will always be a list of lists. The shuffle product is,

$$
H(\vec{a} ; x) H(\vec{b} ; x)=\sum_{\vec{c} \in \vec{a} \amalg \vec{b}} H(\vec{c} ; x) .
$$

This turns the space of polylogarithms into a graded algebra with the grading given by the weight. (The so-called multiple polylogarithms also admit a stuffle product and associated stuffle identities

\footnotetext{
${ }^{1}$ For a recent survey on the different function spaces see [9].

${ }^{2}$ The notation used reflects that of the Mathematica package HarmonicSums [8, 10-13].
} 
which is omitted here for space, see e.g. $[7,8,17]$.) By repeated integration by parts one can also obtain the following class of identities,

$$
H(\vec{a} ; x)=H\left(a_{1} ; x\right) H\left(\vec{a}_{i>1} ; x\right)-H\left(a_{2}, a_{1} ; x\right) H\left(\vec{a}_{i>2} ; x\right)+\ldots+(-1)^{n+1} H\left(a_{n}, a_{n-1}, \ldots, a_{1} ; x\right),
$$

for a weight $n$ polylogarithm.

The relations of the generalized polylogarithms have been extensively studied in Ref. [8]. Furthermore, it is conjectured that the symbol [18-20] provides all relations amongst the generalised polylogarithms. These relations can be used to express physical results numerically more simply however the topic is not yet exhausted. It has been suggested that cyclotomic polylogarithm constants may obey additional relations beyond the shuffle, stuffle and integration by parts identities see [21] for a proper description of the idea.

There are also physics motivations for further study, most notably numerical implementations and PSLQ searches [22] . To calculate the numerical value of a cross-section one must evaluate large numbers of polylogarithms which requires efficient numerical implementation. This in turn also leads to the question of polylogarithms evaluated at 1. This will be explained in Section 2 when the problem examined here is set-up. Secondly, there has been progress in computing arbitrary precision values for amplitudes and using a PSLQ search to fit numerical factors. Such work is helped by further knowledge about the special constants that occur in particle physics.

In this manuscript cyclotomic constants evaluated at 1 are studied. It is explained how up to weight 2 all PSLQ relations can be derived analytically using standard results about classical polylogarithms, which is one of the known results of Ref. [5]. This complements work already present in the literature [5, 23-25] and itself is a part of a larger project [26].

The remainder of the manuscript is organised as follows. In the next section the cyclotomic polylogarithm class is set-up then the set of constants studied are introduced. In Section 3 it is explained how these relations can be calculated and the resulting special numbers are listed. A short summary closes the manuscript.

\section{Cyclotomic polylogarithms}

The $n^{\text {th }}$ cyclotomic polynomial $\Phi_{n}(x)$ may be defined in terms of the primitive roots of unity,

$$
\Phi_{n}(x):=\prod_{\substack{1 \leq k \leq n \\ \operatorname{gcd}(k, n)=1}}\left(x-e^{2 \pi i \frac{k}{n}}\right) .
$$

The letters needed to define cyclotomic polylogarithms [5] are

$$
f_{(k, l)}=\frac{x^{l}}{\Phi_{k}(x)},
$$

so that now the indices of a cyclotomic polylogarithm have two components. The particular values $f_{(1,0)}, f_{(2,0)}$ and $f_{(0,-1)}$ reproduce the usual harmonic polylogarithms (up to sign conventions).

Although the cyclotomic polynomials are defined in terms of roots of unity they give rise to real functions for $x \in(0,1)$ and therefore represent an elegant language for expressing physical results. 
Alternatively, one may forego the advantages of a real representation, and factorise the cyclotomic polynomials leading to complex indices and the (larger class of) generalised polylogarithms. In doing so one gains additional properties such as the scaling property and Hölder convolution which can be useful [27].

For the physical region, $x \in(0,1)$ a Maclaurin series can be used for numerics,

$$
\frac{1}{x-a}=-\frac{1}{a} \sum_{r=0}^{\infty}\left(\frac{x}{a}\right)^{r}
$$

because by (2.1) $\left|\frac{x}{a}\right|<1$ for cyclotomic polylogarithms. After inserting this into the iterated integral expression for a cyclotomic polylogarithm and performing all integrals by expanding everywhere one obtains a series representation. However these series diverge as $x \rightarrow 1$ and therefore have poor convergence for large $x<1$. The solution is to perform a particular variable transformation which expresses a polylogarithm with larger argument $x<1$ in terms of constants and polylogarithms with small argument $x>0$. The formulae are a little lengthy so here a simple example transformation will suffice,

$$
\begin{aligned}
H\left((1,0),(2,0) ; \frac{1-t}{1+t}\right) & =-H((2,0) ; 1) H((2,0) ; t)-H((2,0) ; 1) H(0 ; t)+2 H((2,0),(2,0) ; 1) \\
& +H((2,0),(2,0) ; t)-H((2,0),(1,0) ; 1)+H((0,-1),(2,0) ; t)
\end{aligned}
$$

If $x=\frac{1-t}{1+t}$ then the region $x \in(\sqrt{2}-1,1)$ is mapped to $t \in(0, \sqrt{2}-1)$ thus a cyclotomic polylogarithm at a large argument can be expressed in terms of cyclotomic polylogarithms with small arguments that have faster converging series. One can further exploit a so-called Bernoulli speedup variable which writes the expansion in terms of logarithms of $x$ and gives faster convergence, see e.g. [28]. A consequence of this variable transform is the appearance of several cyclotomic polylogarithms evaluated at 1 . Here these are well-known special values,

$$
H((2,0) ; 1)=\log 2, \quad H((2,0),(2,0) ; 1)=\frac{1}{2} \log ^{2} 2, \quad H((2,0),(1,0) ; 1)=-\mathrm{Li}_{2}\left(\frac{1}{2}\right),
$$

using the dilogarithm, $\mathrm{Li}_{2}$. This new representation (2.4), in terms of the transformed argument $t$, is suitable for efficient numerical representation. For harmonic polylogarithms this does not introduce any significant problems however in the cyclotomic case the constants generated are less well-studied. Indeed at higher weights there are many new special numbers currently given little attention in the literature.

In producing numerical representations for the cyclotomy $k \leq 6$ cyclotomic polylogarithms a large set of cyclotomic constants evaluated at 1 were generated. It was therefore natural to study these numbers and their relations as part of the larger ongoing work of understanding polylogarithms. After eliminating shuffle, stuffle and integration by parts identities there are still many prospective constants. By generating a large number of decimal places of precision for each constant one can try to build a basis by eliminating all PSLQ relations. A similar exercise was conducted in [25]. An important difference is that here the motivation is the constants required for a numerical implementation and this led to the introduction of cyclotomy 12 objects as part of eliminating relations amongst the constants. See [26] for a fuller explanation. Thus the constants studied here include those from the previous studies such as [5,23-25]. Our concern is whether the PSLQ relations can be proven using known properties of polylogarithms. 


\section{PSLQ Relations}

Weight 1 cyclotomic polylogarithms can be expressed in terms of logarithms and $\pi$. Combined with the Lindemann-Weierstraß theorem all relations amongst the cyclotomic polylogarithms can be proven. A small example illustrates the general case,

$$
H((12,3) ; 1)=\int_{0}^{1} d t \frac{t^{3}}{1-t^{2}+t^{4}}=\frac{\pi}{6 \sqrt{3}} .
$$

Due to the introduction of cyclotomy 12 - or equivalently the $12^{\text {th }}$ root of unity if preferred additional numbers beyond those in work [23-25] are found. In Ref. [5] all weight 1 and weight 2 relations have been derived in analytic form.

At weight 2 the direct integrals are fully expressible in terms of logarithms and dilogarithms [5]. Using relations such as those in [3] all weight 2 PSLQ relations can be proven. The first step is direct integration of the polylogarithm which can even be done using many computer algebra systems. Alternatively one may use a well-known result that any weight 2 generalised polylogarithm can be expressed in terms of the dilogarithm and logarithm, see for example [20,27]. Then by factoring cyclotomic polylogarithms into generalised polylogarithms the result follows. The resulting logarithms do not represent further challenges. However the dilogarithms present have complex arguments which can be decomposed into real and imaginary parts using a result by Kummer, as described in Refs. [3,29],

$$
\mathrm{Li}_{2}\left(r e^{i \theta}\right)=\mathrm{Li}_{2}(r, \theta)+i\left[w \log r+\frac{1}{2} \mathrm{Cl}_{2}(2 w)+\frac{1}{2} \mathrm{Cl}_{2}(2 \theta)-\frac{1}{2} \mathrm{Cl}_{2}(2 \theta+2 w)\right] .
$$

Here $w:=\arctan \frac{r \sin \theta}{1-r \cos \theta}$ and $\mathrm{Cl}_{2}$ is Clausen's function. Additionally [3] lists many known particular values for the real part, $\mathrm{Li}_{2}(r, \theta)$. In order to complete the computation and remove all relations it was also required to use two identities due to Ramanujan [30], ${ }^{3}$

$$
\begin{aligned}
\mathrm{Li}_{2}\left(-\frac{1}{3}\right)-\frac{1}{3} \mathrm{Li}_{2}\left(\frac{1}{9}\right) & =\frac{1}{6} \log ^{2} 3-\frac{\pi^{2}}{18}, \\
\mathrm{Li}_{2}\left(\frac{1}{4}\right)+\frac{1}{3} \mathrm{Li}_{2}\left(\frac{1}{9}\right) & =\frac{\pi^{2}}{18}+2 \log 2 \log 3-2 \log ^{2} 2-\frac{2}{3} \log ^{2} 3 .
\end{aligned}
$$

After using all these relations it was possible to arrive at the set of special numbers,

$$
\begin{array}{llll}
\pi, & \log 2, & \log 3, & \log (\sqrt{3}-1), \\
\log (2-\sqrt{3}), & \mathrm{Cl}_{2}\left(\frac{\pi}{3}\right), & \mathrm{Cl}_{2}\left(\frac{\pi}{6}\right), & \operatorname{Li}_{2}\left(\frac{1}{4}\right), \\
\operatorname{Li}_{2}\left(\frac{1}{2}(\sqrt{3}-1)\right), & \operatorname{Li}_{2}\left(\frac{1}{4}(3 \sqrt{3}-5)\right), & \operatorname{Li}_{2}(2-\sqrt{3}), & \operatorname{Li}_{2}(4 \sqrt{3}-7) .
\end{array}
$$

This set includes those numbers found in [23] plus additional numbers from the cyclotomy 12 feature particular to this study. In our analysis we used the packages HarmonicSums [8, 10-13] and Sigma [33,34] to eliminate known relations.

As a corollary of this work it was also possible to prove all the PSLQ relations at weights 1 and 2 conjectured in [25]. In a different, but equivalent, basis representation the corresponding result has been given in [5] in 2011 already.

\footnotetext{
${ }^{3}$ These constants also occur in the calculation of massive 3-loop operator matrix elements $[31,32]$.
} 


\section{Summary}

Although much is known about polylogarithms and their special values at special arguments $x \in \mathbb{C}$ there is no complete theory yet and it has been suggested more may be available to learn. Based on this long term goal a set of specific constants arising from a numerical implementation of cyclotomic polylogarithms were studied. It was possible to show that all relations amongst these constants are already present in the literature and a PSLQ search does not provide any new information for $w \leq 6$ for cyclotomy $k=6$. However, new relations were found for cyclotomy $k=12$. Analytic proofs of part of the relations have been obtained. More details will be presented in Ref. [26]. ${ }^{4}$

\section{References}

[1] E. Remiddi and J.A.M. Vermaseren. Harmonic polylogarithms. Int.J.Mod.Phys., A15:725-754, 2000 [hep-ph/9905237].

[2] J.A.M. Vermaseren. Harmonic sums, Mellin transforms and integrals. Int. J. Mod. Phys. A14:2037-2076, 1999 [hep-ph/9806280];

J. Blümlein and S. Kurth. Harmonic sums and Mellin transforms up to two-loop order. Phys. Rev. D60:014018, 1999 [arXiv:hep-ph/9810241].

[3] L. Lewin. Polylogarithms and associated functions. (North Holland, New York, 1981).

[4] N. Nielsen. Der Eulersche Dilogarithmus und seine Verallgemeinerungen. Nova Acta Leopold. XC Nr. 3: 125-211, 1909.

K.S. Kölbig, J.A. Mignoco, and E. Remiddi. On Nielsen's generalized polylogarithms and their numerical calculation. BIT 10:38-74, 1970.

K.S. Kölbig. Nielsen’s Generalized Polylogarithms. SIAM J. Math. Anal. 17:1232-1258, 1986.

[5] J. Ablinger, J. Blümlein, and C. Schneider. Harmonic sums and polylogarithms generated by cyclotomic polynomials. Journal of Mathematical Physics, 52(10):102301, [arXiv:1105.6063 [math-ph]].

[6] S. Moch, P. Uwer and S. Weinzierl. Nested sums, expansion of transcendental functions and multiscale multiloop integrals. J. Math. Phys. 43:3363-3386, 2002 [hep-ph/0110083].

[7] J. Vollinga and S. Weinzierl. Numerical evaluation of multiple polylogarithms. Comput. Phys. Commun., 167:177-194, 2005 [hep-ph/0410259].

[8] J. Ablinger, J. Blümlein and C. Schneider. Analytic and Algorithmic Aspects of Generalized Harmonic Sums and Polylogarithms. J. Math. Phys. 54:082301, 2013 [arXiv:1302.0378 [math-ph]].

[9] J. Ablinger, J. Blümlein and C. Schneider, Generalized Harmonic, Cyclotomic, and Binomial Sums, their Polylogarithms and Special Numbers, J. Phys. Conf. Ser. 523:012060 (2014) [[arXiv:1310.5645 [math-ph]]].

[10] J. Ablinger. Computer Algebra Algorithms for Special Functions in Particle Physics. PhD thesis, J. Kepler University Linz, April 2012, arXiv:1305.0687 [math-ph].

\footnotetext{
${ }^{4}$ After completion of this paper [35] appeared, in which aspects of bases in the cyclotomic case are discussed under specific conditions.
} 
[11] J. Ablinger, J. Blümlein, C.G. Raab and C. Schneider, J. Math. Phys. 55:112301, 2014 [arXiv:1407.1822 [hep-th]].

[12] J. Ablinger, The package HarmonicSums: Computer Algebra and Analytic Aspects of Nested Sums. PoS (LL2014) 019.

[13] J. Ablinger, J. Blümlein and C. Schneider. Harmonic Sums and Polylogarithms Generated by Cyclotomic Polynomials. J. Math. Phys. 52:102301, 2011 [arXiv:1105.6063 [math-ph]].

[14] K. Chen. Iterated path integrals. Bull. Amer. Math. Soc., 83(5):831-879, 091977.

[15] C. Reutenauer. Free Lie Algebras. (Oxford University Press, Oxford, 1993).

[16] J. Blümlein. Algebraic relations between harmonic sums and associated quantities. Comput. Phys. Commun. 159:19-54, 2004 [hep-ph/0311046].

[17] M. Hoffman. Multiple harmonic series. Pacific J. Math., 152(2):275-290, 1992.

[18] A. B. Goncharov. Galois symmetries of fundamental groupoids and noncommutative geometry. Duke Math. J. 128:209-284, 2005 [arXiv:math/0208144v4 [math.AG]].

[19] C. Duhr. Hopf algebras, coproducts and symbols: an application to Higgs boson amplitudes. JHEP, 08:043, 2012 [arXiv:1110.0458 [math-ph]].

[20] C. Duhr, H. Gangl, and J. Rhodes. From polygons and symbols to polylogarithmic functions. JHEP, 10:075, 2012.

[21] J. Zhao. Standard Relations of Multiple Polylogarithm Values at Roots of Unity. Documenta Mathematica 15:1-34, 2010 [arXiv:0707.1459 [math.NT]].

[22] H.R.P. Ferguson and G.H. Bailey, A Polynomial Time, Numerically Stable Integer Relation Algorithm. RNR Techn. Rept. RNR-91-032, Jul. 14, 1992.

[23] D. J. Broadhurst. Massive three - loop Feynman diagrams reducible to $\mathrm{SC}^{*}$ primitives of algebras of the sixth root of unity. Eur. Phys. J., C8:311-333, 1999 [hep-th/9803091].

[24] M. Kalmykov and B. A. Kniehl. 'Sixth root of unity' and Feynman diagrams: Hypergeometric function approach point of view. Nucl. Phys. Proc. Suppl., 205-206:129-134, 2010 [arXiv:1007.2373 [math-ph]].

[25] J. Henn, A. V. Smirnov, and V. A. Smirnov. Evaluating Multiple Polylogarithm Values at Sixth Roots of Unity up to Weight Six. Nucl. Phys., B919:315-324, 2017 [arXiv:1512.08389 [hep-th]].

[26] J. Ablinger, J. Blümlein, M. Round, and C. Schneider. in preparation.

[27] H. Frellesvig, D. Tommasini, and C. Wever. On the reduction of generalized polylogarithms to $\mathrm{Li}_{n}$ and $\mathrm{Li}_{2,2}$ and on the evaluation thereof. JHEP, 03:189, 2016 [arXiv:1601.02649 [hep-ph]].

[28] T. Gehrmann and E. Remiddi. Numerical evaluation of harmonic polylogarithms. Comput.Phys.Commun., 141:296-312, 2001 [hep-ph/0107173].

[29] A. N. Kirillov. Dilogarithm identities. Prog. Theor. Phys. Suppl., 118:61-142, 1995 [hep-th/9408113].

[30] B. C. Berndt. Ramanujan's Notebooks, Part IV. (Springer, New York, 1994) pp. 323-326.

[31] J. Ablinger, J. Blümlein, C. Raab, C. Schneider and F. Wißbrock. Calculating Massive 3-loop Graphs for Operator Matrix Elements by the Method of Hyperlogarithms, Nucl. Phys. B885:409-447, 2014 [arXiv:1403.1137 [hep-ph]]. 
[32] J. Ablinger, A. Behring, J. Blümlein, A. De Freitas, A. von Manteuffel and C. Schneider. Calculating Three Loop Ladder and $V$-Topologies for Massive Operator Matrix Elements by Computer Algebra. Comput. Phys. Commun. 202:33-112, 2016 [arXiv:1509.08324 [hep-ph]].

[33] C. Schneider. Symbolic Summation Assists Combinatorics. Sém. Lothar. Combin. 56:1-36, 2007, article B56b.

[34] C. Schneider. Simplifying Multiple Sums in Difference Fields. in :Computer Algebra in Quantum Field Theory: Integration, Summation and Special Functions Texts and Monographs in Symbolic Computation eds. C. Schneider and J. Blümlein (Springer, Wien, 2013) pp. 325-360 [arXiv:1304.4134 [cs.SC]].

[35] O. Schnetz. The Galois coaction on the electron anomalous magnetic moment. arXiv:1711.05118 [math-ph]. 